\title{
Arborescences
}

Revue d'études françaises

\section{Susciter le moment critique. De l'investissement de ressources axiologiques à leur mise à distance par des sujets lecteurs élèves}

\section{Marion Sauvaire et Érick Falardeau}

Numéro 6, septembre 2016

Polyphonies : voix et valeurs du discours littéraire

URI : https://id.erudit.org/iderudit/1037507ar

DOI : https://doi.org/10.7202/1037507ar

Aller au sommaire du numéro

Éditeur(s)

Département d'études françaises, Université de Toronto

ISSN

1925-5357 (numérique)

Découvrir la revue

Citer cet article

Sauvaire, M. \& Falardeau, É. (2016). Susciter le moment critique. De l'investissement de ressources axiologiques à leur mise à distance par des sujets lecteurs élèves. Arborescences, (6), 121-147.

https://doi.org/10.7202/1037507ar

\section{Résumé de l'article}

Cet article présente les résultats d'une recherche en didactique du français sur l'enseignement de la lecture littéraire au lycée en France et au cégep au Québec. Nous analysons des interprétations produites par des lecteurs en contexte scolaire dans le cadre de l'étude de La plage des songes (1998), une nouvelle fantastique de Stanley Péan. L'analyse des discours oraux et écrits des élèves est menée selon une approche herméneutique qui valorise la mise à distance réflexive de leur parcours interprétatif par les sujets lecteurs. Nous étudions plus particulièrement les ressources axiologiques mobilisées par les lecteurs en fonction des significations attribuées aux différentes voix énonciatives (la narratrice, les personnages, l'auteur impliqué). Quatre questions sont traitées : de quelle manière les sujets lecteurs attribuent-ils des énoncés aux diverses instances énonciatives ? Cette attribution révèle-t-elle l'investissement de ressources axiologiques (jugements, valeurs, présupposés idéologiques, etc.) ? Attribuer des énoncés à diverses instances contribue-t-il à mettre à distance les ressources axiologiques mobilisées ? Dans quelle mesure les élèves sont-ils capables d'exercer leur réflexivité par rapport aux valeurs, aux opinions, aux présupposés idéologiques qui influencent leurs interprétations et celles de leurs pairs?
Tous droits réservés @ Département d'études françaises, Université de Toronto, 2016
Ce document est protégé par la loi sur le droit d'auteur. L'utilisation des services d'Érudit (y compris la reproduction) est assujettie à sa politique d'utilisation que vous pouvez consulter en ligne. 


\section{SOMMAIRE}

Francis Langevin et Raphaël Baroni.

01 University of British Columbia et Université de Lausanne Introduction

Alain Rabatel. Université de Lyon 1

13 L'énonciation problématisante : en dialogue avec

Le Royaume d'Emmanuel Carrère

Émilie Goin. Université du Luxembourg

39 Analyse d'un discours d'action collective mis en récit.

L'Anarchie dans Les Cloches de Bâle d'Aragon

David Bélanger. Université du Québec à Montréal

54 En contre-jour : la représentation évanescente de l'écrivain dans le roman québécois contemporain

Raphaël Baroni. Université de Lausanne

72 Comment débusquer la voix d'un auteur dans sa fiction?

Une étude de quelques provocations de Michel Houellebecq

Liesbeth Korthals Altes. Rijksuniversiteit Groningen

94 Actes de cadrage, narratologie et herméneutique - à propos de I'indétermination énonciative dans Sujet Angot de Christine Angot

121

Marion Sauvaire et Érick Falardeau. Université Laval

Susciter le moment critique. De l'investissement de ressources axiologiques

Frank Wagner. Université Rennes 2

148 Quand le narrateur boit(e)... (Réflexions sur le narrateur non fiable et/ou indigne de confiance) 


\title{
Susciter le moment critique. De l'investissement de ressources axiologiques à leur mise à distance par des sujets lecteurs élèves
}

\author{
Marion Sauvaire et Érick Falardeau. Université Laval
}

\section{Résumé}

Cet article présente les résultats d'une recherche en didactique du français sur l'enseignement de la lecture littéraire au lycée en France et au cégep au Québec. Nous analysons des interprétations produites par des lecteurs en contexte scolaire dans le cadre de l'étude de La plage des songes (1998), une nouvelle fantastique de Stanley Péan. L'analyse des discours oraux et écrits des élèves est menée selon une approche herméneutique qui valorise la mise à distance réflexive de leur parcours interprétatif par les sujets lecteurs. Nous étudions plus particulièrement les ressources axiologiques mobilisées par les lecteurs en fonction des significations attribuées aux différentes voix énonciatives (la narratrice, les personnages, l'auteur impliqué). Quatre questions sont traitées: de quelle manière les sujets lecteurs attribuent-ils des énoncés aux diverses instances énonciatives? Cette attribution révèle-t-elle l'investissement de ressources axiologiques (jugements, valeurs, présupposés idéologiques, etc.)? Attribuer des énoncés à diverses instances contribue-t-il à mettre à distance les ressources axiologiques mobilisées? Dans quelle mesure les élèves sont-ils capables d'exercer leur réflexivité par rapport aux valeurs, aux opinions, aux présupposés idéologiques qui influencent leurs interprétations et celles de leurs pairs?

Le moment critique ou « la critique des idéologies » est le détour nécessaire que doit prendre la compréhension de soi, si celle-ci doit se laisser former par la chose du texte et non par les préjugés du lecteur.

(Ricœur $1986: 131$ )

Cet article propose une analyse de discours d'élèves qui prolonge une recherche doctorale sur l'enseignement de la lecture littéraire (Sauvaire 2013). Il se situe dans les domaines des études littéraires et de la didactique du français et traitera plus spécifiquement des interprétations produites par des lecteurs réels en contexte scolaire (lycée en France et cégep au Québec) dans le cadre de l'étude de La plage des songes (1998), une nouvelle fantastique de Stanley Péan. L'analyse sera menée sous l'angle des rapports entre la polyphonie énonciative construite par le texte littéraire et les ressources axiologiques mobilisées par les lecteurs pour attribuer des significations aux différentes voix énonciatives (la narratrice, les personnages, l'auteur impliqué). La visée de cette analyse est compréhensive et non praxéologique. Nos 
objectifs sont de décrire comment les élèves relient certains énoncés à différentes instances narratives, en quoi ce phénomène d'attribution rend perceptibles certaines ressources axiologiques mobilisées pour interpréter le texte. In fine nous souhaitons comprendre comment les élèves mettent à distance les présupposés idéologiques et axiologiques qui influencent leurs interprétations.

Les concepts de lecture littéraire et de sujet lecteur, ainsi que la notion de ressources subjectives seront d'abord brièvement définis selon une approche théorique inspirée de l'herméneutique de Paul Ricœur. Nous présenterons ensuite les quatre questions d'analyse retenues pour cet article, ainsi que les grandes lignes de l'étude de cas menée dans deux classes. Une présentation de l'œuvre précèdera l'analyse des discours des lecteurs en formation.

\section{Enseigner la lecture littéraire}

Ces dix dernières années, dans le domaine de l'enseignement de la lecture littéraire, de nombreuses recherches ont encouragé ${ }^{1}$ la prise en compte théorique et pratique de l'activité des sujets lecteurs.

\subsection{Former des sujets lecteurs}

Dans le domaine de la didactique du français, et plus précisément en ce qui concerne l'enseignement de la littérature, de nombreuses recherches ont contribué à élaborer le concept de lecture littéraire (Daunay 2007 ; Dufays et al. 2005 ; Dufays 2013 ; Gervais et Bouvet 2007 ; Louichon 2011 ; Reuter 1995; Rouxel 2002 ; Tauveron 2002). Ce concept repose sur deux postulats, faisant consensus parmi les chercheurs, à savoir que le texte littéraire est par nature "inachevé » (Ricœur 1985) et qu'il est constitué de " lieux d'indétermination" (Iser 1971, 1985) que seul le lecteur est en mesure de combler pour reconfigurer les significations. Ce concept de lecture littéraire se situe dans la continuité des théories de la réception ayant contribué à revaloriser l'activité lectorale, notamment sous les figures du "lecteur implicite» d'Iser (1985) ou du «lecteur modèle» d'Eco (1985). Toutefois, dans son usage particulier en didactique du français, le concept s'éloigne de ces modèles dans la mesure où la lecture est conçue non seulement comme une activité prévue par le texte, participant à la construction d'une figure abstraite d'un lecteur "impliqué » (Ricœur 1985 : 302), mais surtout comme l'activité de sujets réels situés dans des contextes

\footnotetext{
${ }^{1}$ Ce texte adopte l'orthographe rectifiée.
} 
d'interaction particuliers, les situations scolaires. Plusieurs recherches menées sur l'activité des "sujets lecteurs » (Rouxel et Langlade 2004 ; Louichon 2009 ; Mazauric, Fourtanier et Langlade 2011a) ont contribué à faire des diverses interprétations qu'ils produisent un objet privilégié de la recherche en didactique (Dufays 2007 ; Langlade et Fourtanier 2007).

Le concept de sujet lecteur permet de reconnaitre que l'activité interprétative repose sur l'investissement subjectif de chaque lecteur. L'investissement subjectif ne se résume en aucun cas à la somme des réactions affectives, mais il est entendu ici comme la mobilisation par le lecteur d'un grand nombre de ressources (ses connaissances, ses habiletés cognitives, ses expériences, ses valeurs, ses représentations sociales, ses présupposés idéologiques, etc.) pour produire une interprétation du texte littéraire qui soit recevable aux yeux des membres de sa communauté interprétative (Fish 2009 ; Citton 2007). En effet, selon nous, la reconnaissance du caractère subjectif de l'interprétation (comme processus et comme produit de la lecture) implique de reconsidérer les critères de validation des interprétations : on passe d'un critère de vérité, la prétendue objectivité du sens textuel, à un critère d' "acceptabilité ", construit de manière intersubjective sur la base de connaissances, de procédures, de valeurs partagées par une communauté interprétative.

\subsection{De l'hétérogénéité énonciative à la diversité interprétative}

Dans ce contexte, la lecture apparait d'abord comme un espace dialogique, celui de la rencontre entre "le monde du texte » et le "monde du lecteur» (Ricœur 1986), où l'hétérogénéité énonciative propre au récit fait l'objet de multiples reconfigurations narratives par les lecteurs. Ces reconfigurations narratives, plurielles, changeantes et parfois contradictoires, apparaissent comme les traces de l'investissement du sujet dans l'acte de lire. Or, cet investissement subjectif peut faire l'objet d'une mise à distance réflexive, d'une "distanciation de soi à soi " (Ricœur 1985) ${ }^{2}$. Selon cette approche herméneutique, la subjectivité se révèle transformée, de manière réflexive et critique, au terme du processus de la compréhension des textes, en particulier des récits (Ricœur 1990).

En classe, la lecture apparait ensuite comme un espace intersubjectif dans la mesure où les diverses interprétations doivent être partagées,

\footnotetext{
${ }^{2}$ Selon Ricœur, le concept herméneutique de distanciation recouvre le geste second par lequel un sujet met à distance son appartenance au monde. Dans le cadre de l'interprétation des textes, la distanciation est au cœur même de l'appropriation. «Parce que la distanciation est un moment de l'appartenance, la critique des idéologies peut être incorporée, comme un segment objectif et explicatif, dans le projet d'élargir et de restaurer la communication et la compréhension de soi » (Ricœur 1985 : 57).
} 
confrontées, évaluées. Ces deux espaces, dialogiques et intersubjectifs, se présupposent mutuellement : d'une part, l'acte de lecture individuel révèle ou actualise, de manière éminemment singulière, la polysémie textuelle; d'autre part, c'est dans la mesure où le "lecteur participe aux attentes sédimentées dans le public qu'il est constitué en lecteur compétent » (Ricœur 1985 : 304). Il convient encore de considérer que non seulement différents lecteurs peuvent formuler différentes interprétations, mais aussi que chaque lecteur est capable de produire plusieurs interprétations, au fur et à mesure de ses relectures et de ses échanges avec ses pairs et l'enseignant. Nous appelons "diversité interprétative " l'ensemble des interprétations produites concomitamment par différents lecteurs en interaction et successivement par un même lecteur. Dans le cadre de cette recherche, nous avons exploré les médiations entre l'apprentissage de la diversité interprétative et la compréhension réflexive de leur diversité par les sujets en formation. Si la diversité interprétative nous apparait comme une médiation privilégiée de la compréhension de la diversité subjective, c'est notamment parce que les diverses ressources subjectives mobilisées par les lecteurs déterminent en partie la production et l'évolution de leurs interprétations.

\subsection{Les diverses ressources subjectives du lecteur}

L'expression "ressources subjectives" (Sauvaire 2013) renvoie à l'ensemble des connaissances, des procédures, des valeurs, des représentations et des expériences dont dispose le lecteur pour interpréter le texte. Les ressources sont qualifiées de subjectives en cela qu'elles font l'objet d'une mobilisation, plus ou moins consciente, par le sujet lecteur, et ce, qu'elles soient le fruit de l'intériorisation de conduites sociales, de l'appropriation de savoirs ou d'expériences plus singulières. Elles constituent un réseau mouvant d'éléments très fortement interconnectés qu'il n'est possible d'observer que dans les discours que les lecteurs tiennent a posteriori sur leur lecture. L'analyse des diverses ressources mobilisées permet, selon nous, d'approfondir significativement la compréhension du caractère subjectif de l'interprétation ; elle ne peut toutefois prétendre constituer un modèle d'explication du processus interprétatif, puisqu'elle n'en est qu'une composante.

En nous inspirant de l'idée d'une typologie des «sources de l'activité fictionnalisante » proposée par Langlade et Fourtanier (2007), nous avons dégagé théoriquement six ensembles de ressources : épistémiques, cognitives, psychoaffectives, socioculturelles, axiologiques et matérielles / spatiotemporelles. Chacun de ces ensembles constitue une des catégories d'analyse qui ont ensuite été divisées en sous-catégories à partir de l'analyse des entretiens et des écrits des élèves.

1) Les ressources épistémiques regroupent sept catégories de connaissances: les connaissances générales sur la littérature (liées, par 
exemple, à l'histoire littéraire, au fonctionnement du champ littéraire, à l'intertextualité, à la théorie littéraire) ; les connaissances utiles à l'analyse textuelle; les connaissances concernant les contextes de production et les auteurs ; les connaissances procédurales (c'est-à-dire des savoir-faire disciplinaires) ; les réseaux de textes (par exemple ; la connaissance de textes reliés entre eux par le thème, l'appartenance générique, les procédés stylistiques, sans nécessairement de rapports intertextuels explicites) ; les connaissances spécifiques à la dimension fictionnelle; et finalement les connaissances linguistiques (phonologiques, orthographiques, syntaxiques, sémantiques et pragmatiques).

2) Les ressources cognitives regroupent cinq catégories: les microprocessus, qui concernent le niveau de la phrase (reconnaissance des mots; lecture par groupe de mots) et les processus d'intégration entre les phrases (utilisation des référents, des connecteurs) (Giasson 1990); les macroprocessus, qui concernent l'identification des idées principales, de la macrostructure du texte; les processus d'élaboration, qui sont des processus d'inférence non prévus par le texte tels que l'activation d'images mentales à travers lesquelles le lecteur se représente l'action; les stratégies cognitives et métacognitives, qui constituent des répertoires de moyens dans lesquels puise le lecteur pour repérer, classer, synthétiser des éléments d'informations, planifier sa lecture, etc.

3) Les ressources psychoaffectives désignent les expériences personnelles que le lecteur met en écho avec le texte pour construire du sens; les émotions et les sensations que suscite le texte lu; les phénomènes d'identification du lecteur avec le personnage.

4) Les ressources axiologiques font référence aux jugements de valeur que porte le lecteur quant aux motivations, aux actions, aux énoncés qu'il attribue à un personnage ou au narrateur ; aux croyances, aux systèmes de valeurs et aux discours idéologiques que le lecteur relie explicitement aux énoncés ; à la recherche de comportements exemplaires.

5) Les ressources socioculturelles regroupent les appartenances à un ou plusieurs groupes culturels (régionaux, nationaux, religieux); les représentations de la diversité culturelle, en termes de différenciation, d'acceptation ou de rejet par exemple; la prise de position par rapport à des conflits communautaires comme le racisme, l'intolérance religieuse; les stéréotypes culturels par rapport à des groupes, des pratiques, des classes sociales, etc.; l'appartenance à des groupes de lecteurs scolaires ou non scolaires qui influencent la façon d'appréhender des textes; l'influence de passeurs culturels (enseignants, parents, animateurs culturels, etc.) ; les liens avec des pratiques culturelles éclectiques sur le plan de leur légitimation (Lahire 2004).

6) Les ressources matérielles et spatiotemporelles font référence aux supports concrets de lecture (par exemple, des supports imprimés ou électroniques, des illustrations, la facture de l'ouvrage) et à la situation de 
lecture dans le temps et l'espace (privé ou public).

Concernant les ressources, les principaux résultats de Sauvaire (2013) tendent à montrer que la diversité des interprétations subjectives dépend en partie de la nature des ressources mobilisées, de leur nombre et des liens, implicites ou explicites, que le sujet lecteur établit entre elles. Cette analyse apporte ainsi un éclairage nouveau sur le développement des compétences interprétatives et les éventuelles difficultés des élèves. Par exemple, les élèves qui produisent plusieurs interprétations riches et complexes sont ceux qui mobilisent des ressources de différents types et qui font des liens entre, par exemple, d'une part, des ressources socioculturelles et psychoaffectives, et d'autre part, des stratégies de lecture et des savoirs disciplinaires. Inversement, quand l'interprétation du texte est partielle, voire erronée, un seul type de ressources a été mobilisé - ce qui pourrait expliquer les difficultés de certains élèves à formuler des interprétations qui rendent compte de la complexité du texte. Par ailleurs, l'analyse des ressources subjectives nous renseigne sur l'investissement des lecteurs tout au long de leur parcours interprétatif. En effet, des élèves peu investis subjectivement au début de la séquence didactique ont progressivement mobilisé de nouvelles ressources, en particulier après les activités collaboratives (comités de lecture et débats interprétatifs). Ces résultats nous encouragent à approfondir l'hypothèse selon laquelle la subjectivité s'élabore dans l'acte de lecture. Loin d'être un donné, antérieur et extérieur à l'acte de lecture, l'investissement subjectif pourrait ainsi faire l'objet d'une intervention didactique.

\section{Problématique}

Dans le cadre de cet article, nous analysons plus spécifiquement les rapports entre la polyphonie énonciative et la mobilisation de ressources axiologiques par les élèves. Quel est le rôle de ces ressources axiologiques dans l'activité des lecteurs? Cette question apparait d'autant plus importante que les ressources axiologiques sont susceptibles d'influencer grandement l'appréciation critique du texte lu et que cette compétence appréciative fait l'objet d'évaluations certificatives de fin de cycle très fortement valorisées par l'institution scolaire 3 . Or, en classe, la diversité des systèmes idéologiques, des valeurs et des croyances est souvent analysée en termes de représentations construites par le texte, mais elle est rarement rapportée à la diversité des lectures qui peuvent en être faites (Daunay et al. 2009). Pourtant, nous

\footnotetext{
${ }^{3}$ En témoigne la pérennité de la dissertation littéraire qui reste l'exercice prescrit pour la certification de fin d'études pré-universitaires, qu'il s'agisse de l'épreuve du baccalauréat en France ou de l'Épreuve uniforme de français au Québec.
} 
pensons que la polyphonie engage directement l'interprète et exige un positionnement, non seulement discursif mais idéologique. La polyphonie semble engager le lecteur à mobiliser des ressources axiologiques pour attribuer des significations provisoires aux divers énoncés. L'élaboration de ces significations dépend-elle des instances énonciatives auxquelles ces énoncés sont attribués par les lecteurs? Il parait difficile de clarifier dans quelle mesure les ressources axiologiques déterminent cette attribution, et ce, d'autant plus qu'il s'agit de lecteurs en formation. En effet, l'interprétation des différentes voix énonciatives et la mise au jour des cadres normatifs qui président à cette interprétation sont le plus souvent le fait de lecteurs avertis, sinon experts. De telles habiletés interprétatives et réflexives peuvent-elles être observées dans les discours des sujets lecteurs en formation? Pour répondre à cette question, nous analysons les discours écrits et oraux produits par des élèves au sujet de leur lecture. S'il nous paraît utile de décrire comment la mobilisation de ressources axiologiques influence les interprétations proposées par les élèves, cela nous semble insuffisant, dans la mesure où, selon nous, l'enseignement de la lecture littéraire devrait non seulement contribuer à la subjectivation de l'expérience lectorale mais aussi à sa mise à distance réflexive. Autrement dit, nous essayons de mieux comprendre comment des sujets lecteurs en formation prennent conscience réflexivement du caractère subjectif de leur lecture, et en particulier des présupposés idéologiques et axiologiques qui influencent leurs interprétations.

Notre étude poursuit donc deux objectifs principaux :

1. Décrire comment les élèves relient assez spontanément certains énoncés à différentes instances narratives (auteur impliqué, narrateur, personnage) et comment ce phénomène d'attribution rend perceptibles certaines ressources axiologiques et leur influence sur l'élaboration de significations par les lecteurs.

2. Comprendre comment les élèves mettent ou non à distance les présupposés idéologiques et axiologiques qui influencent les interprétations.

L'analyse sera guidée par les quatre questions suivantes :

1. De quelle manière les sujets lecteurs attribuent-ils des énoncés aux diverses instances énonciatives?

2. Comment décrire les ressources axiologiques (jugements, valeurs, présupposés idéologiques, etc.) mises en évidence par cette attribution ?

3. De quelle façon l'attribution des énoncés à diverses instances contribue-t-elle à mettre à distance les ressources axiologiques mobilisées ?

4. Dans quelle mesure les élèves sont-ils capables d'exercer leur réflexivité par rapport aux valeurs, aux opinions, aux présupposés idéologiques qui influencent leurs interprétations et celles de leurs pairs? 


\section{Quelques précisions d'ordre méthodologique}

Cet article constituant un prolongement de la recherche doctorale de la première auteure (Sauvaire 2013), nous en présentons sommairement la méthodologie ${ }^{4}$ et nous concentrons sur les données sélectionnées pour la présente analyse. Nous avons élaboré une séquence didactique ${ }^{5}$ que deux enseignants ont expérimentée, l'un avec une classe de lycée en France (seconde) et l'autre avec une classe de cégep au Québec (cours 102) ${ }^{6}$. La séquence didactique, d'une durée de trois semaines, était axée sur l'articulation entre des activités de lecture, d'écriture et d'oral. Elle favorisait un va-et-vient entre la production d'interprétations singulières à l'écrit et la confrontation des interprétations lors d'activités collaboratives à l'oral (par exemple, des comités de lecture, un débat interprétatif). L'expérimentation de cette séquence nous a permis de recueillir de nombreuses données qualitatives concernant la production de diverses interprétations par les élèves, et ce, à l'aide de plusieurs instruments complémentaires: des textes d'élèves rédigés tout au long de la séquence, des enregistrements vidéos des cours, des enregistrements audios des activités collaboratives, des entretiens d'explicitation avec les élèves, des entretiens semi-dirigés avec les enseignants et des notes d'observation de la chercheuse.

L'approche méthodologique retenue est l'étude multi-cas et multisites (Miles et Huberman 2003) qui permet de recueillir et d'analyser un grand nombre de données qualitatives sur un petit nombre de participants en valorisant leur situation dans des contextes spécifiques. Dans cet article, nous analysons principalement les récits de lecture écrits immédiatement après une première lecture individuelle de l'œuvre et les textes réflexifs rédigés à la fin de la séquence par les élèves dans le but de mettre à distance rétrospectivement l'évolution de leurs interprétations. Ces textes ont servi de support lors d'entretiens d'explicitation (Vermersch 1991, 2004) menés avec un échantillon de sept élèves au terme de la séquence. Les transcriptions de ces entretiens sont également analysées.

Le codage des données pour l'analyse de contenu a été réalisé de façon semi-inductive à partir de catégories élaborées théoriquement, mais précisées au long du processus d'analyse (comme nous l'avons illustré ci-dessus avec l'exemple des ressources subjectives). L'interprétation des données repose sur la présentation de "parcours de lecteurs ", qui sont des synthèses narratives et

\footnotetext{
${ }^{4}$ Pour une description du protocole de collecte et d'analyse des données voir aussi Sauvaire (2015).

${ }^{5}$ Une séquence est une suite coordonnée d'activités visant les mêmes apprentissages (Simard et al. 2010).

${ }^{6} \mathrm{La}$ recherche ayant été réalisée dans le cadre d'une cotutelle internationale, nous avons pu mettre en ouvre le dispositif de collecte de données dans deux pays francophones. Toutefois, la recherche, de nature qualitative, n'a aucune prétention comparative.
} 
analytiques des données propres à chaque sujet lecteur, dans lesquels nous avons replacé les discours des élèves (préalablement codés et analysés) dans le contexte de leurs interactions et dans l'ordre chronologique de leur formulation. Selon nous, ce type de présentation des données permet de valoriser le sens que les sujets lecteurs donnent à leur activité, dans le cas présent, à leur parcours interprétatif. Pour cet article, nous avons procédé à une analyse plus fine des parcours de lecteur à l'aune des quatre questions présentées dans la problématique.

\section{Analyse de la nouvelle à l'étude : La plage des songes}

Avant d'interpréter les résultats, il nous semble essentiel de présenter succinctement l'œuvre littéraire étudiée. La lecture proposée se concentre sur les enjeux interprétatifs qui font l'objet des discours des élèves lecteurs. Par souci de concision, certains axes d'études ont donc volontairement été mis de côté.

\subsection{Résumé de La plage des songes}

La plage des songes, première nouvelle d'un recueil éponyme de Stanley Péan paru en 1988, réactualise le topos fantastique de l'inversion de la réalité et du rêve, dans le contexte particulier de l'immigration haïtienne au Québec. Le récit est centré sur la relation entre deux migrants d'origine haïtienne Évelyne, une jeune documentaliste, et Christian, un élève de sept ans - à Chicoutimi, au Québec. Évelyne prend rapidement l'enfant sous son aile, le protégeant de la violence raciste de ses camarades d'école. Elle lui fait découvrir l'histoire, la langue et la gastronomie haïtiennes et lui raconte des contes créoles. Christian est un être énigmatique, à la peau noire et aux yeux bleus, qui semble posséder le don mystérieux de donner corps et vie aux images du passé. Progressivement, ils se transportent tous les deux à Montruis, sur la plage d'enfance d'Évelyne, en Haïti. Cependant, ces allers et retours de plus en plus fréquents vers la plage des songes réveillent les spectres du passé et provoquent la colère d'Alceste, le père de Christian. Le soir d'Halloween, lors d'une fugue, Christian est roué de coups et laissé pour mort par d'autres enfants. À travers cette épreuve, Évelyne remet en cause son obsession de l'origine et fait le deuil de son passé. Dans l'épilogue, elle retrouve Christian quinze ans plus tard. Il semble avoir perdu sa magie ancienne, mais il est devenu un peintre talentueux, qui a ouvert une école pour les jeunes artistes de la diaspora, grâce à l'appui de son père. 


\subsection{La poétique de l'errance ou le renouvèlement des figures de la migration}

Pour saisir l'originalité de cette nouvelle de Stanley Péan par rapport aux récits publiés au Québec ces quarante dernières années par des écrivains d'origine caribéenne, nous suggérons de cerner brièvement l'évolution de la figure du migrant à travers trois poétiques : l'exil, la migrance et l'errance.

L'exil est une expérience exacerbée de l'arrachement à la terre natale qui, paradoxalement, accentue la prégnance du territoire. L'impossibilité du retour semble renforcer la quête fantasmée de l'origine, dont le souvenir s'efface avec le temps. L'exilé est condamné à la dislocation, il ne peut combler la distance entre le soi et l'autre, l'ici et l'ailleurs, le présent et le passé. Pour la première génération d'écrivains exilés, l'obsession de l'origine et le sentiment d'une dépossession identitaire primaient la relation à l'altérité québécoise?.

Progressivement, ce devoir de mémoire est transformé par la confrontation avec le présent québécois. Le pays d'accueil est représenté, notamment la ville, qui est explorée par le personnage migrant. En arpentant le lieu de l'autre, l'écrivain migrant en fait son espace d'énonciation, mais il altère aussi l'imaginaire québécois. La migrance des écrivains a pour corrélat la migration des imaginaires, la dissémination des repères identitaires, la contamination des espaces. Il ne faudrait pas hâtivement en conclure à une hybridation sans heurts des cultures et des identités. Ces récits mettent en scène des espaces juxtaposés et des discours contradictoires qui coexistent sans forcément s'harmoniser. Dans ces récits, l'expérience du retour au pays natal est rendue possible grâce à la chute ou à l'affaiblissement des régimes dictatoriaux. Si, dans l'exil, tout retour était exclu, dans la migrance, le retour est envisageable mais profondément désenchanté. L'image du pays d'origine, mythifiée par la nostalgie exilique, ne correspond plus au pays réel que redécouvre le migrant. La distance entre le pays d'origine et le pays d'exil cède le pas à une nouvelle tension entre le pays rêvé, cristallisé dans la mémoire et les récits de la diaspora, et le pays réel. Devenu un étranger chez lui sans être tout à fait enraciné ailleurs, l'écrivain migrant déploie progressivement son écriture à partir des hétérotopies (Foucault 2009), ces zones de contacts entre des espaces hétérogènes. Cela lui permet d'élargir son lieu d'énonciation et de se jouer des frontières territoriales ${ }^{8}$.

\footnotetext{
${ }^{7}$ Voir par exemple Ollivier, É. (1977). Paysage de l'aveugle. Montréal : Pierre Tisseyre; Phelps, A. (1976). Mémoire en colin-maillard. Montréal : Nouvelle optique; Étienne, G. (1974). Le nègre crucifié. Montréal : Nouvelle optique.

${ }^{8}$ L'œuvre romanesque d'Émile Ollivier illustre bien ce passage de l'exil à la migrance : que l'on compare, par exemple, Le paysage de l'aveugle (1977) et Passage (1991).
} 
La "pensée de l'errance» (Glissant 1990) est l'exploration par l'écriture d'un tiers espace (Bhabha 2007) qui permet d'assumer les déséquilibres entre le présent et le passé, l'ici et l'ailleurs, l'expression d'un " je " et celle d'un "nous». Cet espace interstitiel est créé par la mise en relation de lieux autrefois juxtaposés. Ainsi, le pays réel et le pays rêvé s'imbriquent dans l'imaginaire des personnages ou se déplacent au gré d'une narration polyphonique, dans un constant aller-retour entre le pays natal et le pays d'accueil. En effet, l'expérience du désenchantement ne signe pas l'abandon du questionnement sur le retour au pays, comme en témoigne L'énigme du retour de Dany Laferrière ou La plage des songes de Stanley Péan. Cependant, ces écrivains privilégient désormais la dimension fantasmagorique et symbolique du retour. La tension entre le pays rêvé et le pays réel devient l'objet d'une fiction : le pays intérieur. Ce pays intérieur est construit comme un espace interstitiel : espace liminal entre la vie et la mort dans Pays sans chapeau de Laferrière, espace intermédiaire entre l'ici et l'ailleurs dans L'énigme du retour, ou encore, tiers-espace entre le rêve et le réel dans La plage des songes de Péan. À la différence des exilés, pour ce dernier, le pays d'origine n'est pas perdu : il est fictif. Ce pays intérieur est en grande partie reconstruit par l'acte d'écriture, dont il devient un enjeu central.

Si Stanley Péan se présente comme un « voleur de mémoires " (Olivier 1998), c'est parce qu'il recompose son Haïti intérieure à partir des matériaux épars empruntés aux souvenirs et aux récits des migrants qui l’ont précédé. Le caractère composite et hétérogène du pays intérieur est le fruit de la mise en relation de divers discours parfois contradictoires. Ainsi, nombreux sont les récits enchâssés, qui permettent de faire entendre plusieurs voix narratives et de composer une image mobile et plurielle du pays intérieur. Cette hétéroglossie remet en cause toute recherche totalisatrice du sens, toute appréhension univoque de l'identité. Par exemple, l'auteur utilise le procédé de la mise en abime, en insérant des contes dans le récit-cadre. Ce procédé lui permet de s'inscrire dans la tradition orale du conte créole, tout en interrogeant la réception parfois problématique de ces récits dans le contexte nouveau de la migration. Le pays d'origine s'en trouve doublement construit : il est une fiction, elle-même construite à partir des multiples fictions transmises et réinterprétées par les membres de la diaspora. L'originalité de $L a$ plage des songes réside dans la création de cet espace interstitiel entre le pays réel et le pays rêvé, à la jonction de l'ici et de l'ailleurs, de la mémoire exilique et du présent de l'enracinement.

\subsection{Hétérogénéité énonciative et hétérotopie : de quelques problèmes interprétatifs}

Outre sa brièveté, cette nouvelle a l'avantage de susciter de nombreux problèmes interprétatifs dont la résolution constitue un défi à la portée des 
élèves. Certains génèrent des questionnements éthiques et esthétiques susceptibles de favoriser l'investissement de ressources axiologiques par les lecteurs: il s'agit de la représentation des conflits communautaires, en particulier la violence raciste ; de l'inversion du rêve et de la réalité qui plonge nécessairement l'interprète dans l'indécision quant à la nature du don de Christian et quant à la crédibilité de la narratrice; de la mort de Christian annoncée textuellement dès l'incipit, mais dont la dimension symbolique ne peut être perçue qu'à travers l'élucidation de certaines métaphores.

\subsubsection{Les conflits engendrés par la migration}

Dans La plage des songes, Péan décrit la violence raciste de certains enfants québécois envers le jeune Haïtien. Deux scènes de violence très crues encadrent le récit rétrospectif de la relation entre Évelyne et Christian. Cependant, contrairement à ce qu'on observe dans d'autres nouvelles de Péan, les conflits entre les communautés ou les errements du modèle multiculturaliste canadien n'y sont pas au centre du propos. Le conflit entre les personnes de couleur différente, qui est très présent dans les discours culturalistes nord-américains, est dépassé au profit d'une réflexion sur les disparités individuelles au cour même de la communauté haïtienne du Québec. À travers la tension entre Évelyne et Alceste, le père de Christian, l'auteur remet en cause une opposition simpliste entre le modèle du parvenu assimilé et celui de l'exilé enfermé dans le ressassement de l'arrachement à son ile natale. Cette opposition, qui structure fortement les discours et les comportements au sein de la diaspora, laisse penser que les lignes de fractures à l'intérieur d'une même communauté sont parfois plus contraignantes que les frontières entre des communautés différentes.

Ainsi, le jeune Christian est disloqué entre deux figures tutélaires: Alceste, le père biologique, et Évelyne, la mère de substitution. Alceste Marcellin apparait d'abord comme le stéréotype du bourgeois assimilé à la culture d'accueil et remarié à une Québécoise. Selon la narratrice, il est «typique d'une certaine classe d'intellectuels haïtiens. Professeur d'histoire du Québec, il cultivait un accent français qui masquait mal son intonation chantante d'Antillais " (1998: 15). Il est affublé du surnom de Mysie Oreo ${ }^{9}$ par Évelyne et son frère, Edgar, qui se posent en représentants de la diaspora communautaire, attachés à la tradition créole et perpétuant leur culture d'origine dans tous les aspects de la vie quotidienne (la musique, la langue, la cuisine, le fonctionnement familial, etc.). Pour Christian, l'écart se creuse entre la réalité du présent québécois, dominé par l'autorité du père et l'ostracisme des camarades, et une Haïti rêvée, fantasme issu

${ }^{9}$ Les « Oreos " sont des petits gâteaux chocolatés fourrés à la crème, ils sont noirs à l'extérieur et blancs à l'intérieur. Il s'agit d'une insulte visant à se moquer d'une attitude jugée assimilationniste et masquant un complexe de " colonisé ». 
de la nostalgie d'Évelyne. Christian tente de résoudre cette dislocation, à la fois géographique et symbolique, au travers d'un déplacement continuel entre les deux espaces. De ce déplacement nait une hétérotopie: Christian crée depuis sa chambre un espace imaginaire où se superposent le passé et le présent, l'ici et l'ailleurs, le pays réel et le pays rêvé. Cette hétérotopie est rendue possible grâce à l'intervention du fantastique. Dans La plage des songes, l'enfant est le porteur du fantastique : il possède un don qui lui permet de faire exister un espace soustrait à la violence $\mathrm{du}$ réel à partir des souvenirs de la narratrice. L'irruption du fantastique pourrait souligner ici l'effort d'un individu pour dépasser l'alternative intenable entre l'assimilation et l'exil.

\subsubsection{Le fantastique : inversion du rêve et de la réalité}

Les caractéristiques du genre fantastique engagent le lecteur à remettre en cause ses conceptions familières du temps, de l'espace et de la causalité. Roger Caillois propose de définir le fantastique comme "l'irruption de l'inadmissible " dans un monde soumis à une causalité rigoureuse et à une temporalité et une spatialité familières ${ }^{10}$. Selon Caillois, bien que rare et difficile à appréhender, le thème de l'inversion du rêve et de la réalité est particulièrement représentatif du fantastique du fait du renversement total qu'il cherche à faire admettre. Ce renversement a pour conséquence une autre caractéristique du fantastique : la difficulté, voire l'impossibilité, pour le narrateur de trancher entre deux interprétations $\mathrm{du}$ phénomène surnaturel. Cette indécidabilité propre au fantastique, mise en lumière par Tzvetan Todorov dans l'Introduction à la littérature fantastique, concerne également le lecteur. Cette indécision de l'interprète constitue la caractéristique qui distingue le fantastique des registres proches que sont l'étrange et le merveilleux.

Dans La plage des songes, le don que possède Christian de rendre leur matérialité aux objets, aux paysages et aux spectres à partir de la mémoire d'autrui constitue l'élément fantastique central. Le lecteur hésite entre des interprétations " rationnelles" (par exemple : Christian est un enfant à l'imagination débordante et Évelyne est absorbée dans sa nostalgie, ils finissent par ne plus distinguer le réel de leurs désirs) et des interprétations "surnaturelles » (par exemple : Christian est un magicien, voire un sorcier vaudou, capable de matérialiser les rêves, ou bien, c'est la réalité qui est un songe). Or, dans le registre fantastique, le fait que le

\footnotetext{
10 "L'épouvante propre au conte fantastique sévit seulement en un monde incrédule, où les lois de la nature sont tenues pour inflexibles et immuables. Elle y apparait comme la nostalgie ou la menace d'un univers accessible aux puissances des ténèbres et aux émissaires de l'audelà. En outre, préfiguration d'une autre espèce de récits, le temps s'y dédouble ou s'y multiplie, l'espace y connait d'étranges vides, des territoires interdits et sans étendue, des " poches " insituables. La causalité enfin subit en ces parages d'inexplicables injures " (article « littérature fantastique » de l'Encyclopédie Universalis, p. 27).
} 
lecteur puisse attribuer des significations contradictoires aux différentes voix narratives va acquérir une importance particulière, puisque de cette attribution dépendra la crédibilité accordée à la voix narrative par l'interprète. Du fait même de sa confusion, le point de vue de la narratrice peut apparaitre comme oblitéré ou biaisé, ce qui peut conduire les lecteurs à privilégier le point de vue de personnages secondaires, jugés plus crédibles.

\section{Résultats : analyse des discours de sujets lecteurs en formation}

Tel que nous l'avons annoncé dans la problématique, l'analyse des extraits des discours d'élèves est structurée autour de quatre questions. Nous voyons d'abord comment les lecteurs attribuent des énoncés à différentes instances énonciatives, puis en quoi cette attribution révèle certaines de leurs ressources axiologiques et comment les décrire. Nous analysons ensuite la mise à distance de ces ressources axiologiques et enfin, plus largement, le développement de la réflexivité des lecteurs en formation.

\subsection{De quelle manière les sujets lecteurs attribuent-ils des énoncés à diverses instances énonciatives?}

Pour construire du sens, les élèves relient assez spontanément des énoncés à des instances qui en seraient responsables, l'auteur au premier rang. Comment expliquer cette valorisation de l'instance auctoriale dans les discours des lecteurs en formation?

\subsubsection{Prégnance de l'intention auctoriale}

À première vue, la persistance d'une confusion entre les notions de narrateur et d'auteur chez certains lecteurs plus en difficulté apparait comme une explication plausible, mais elle s'avère insuffisante dans la plupart des cas. En effet, le fait d'accorder une dimension autobiographique au récit permet souvent aux jeunes lecteurs d'investir davantage de ressources psychoaffectives ou axiologiques, ce qui favorise leur identification avec les personnages. Lucas affirme par exemple: "Je me sens proche de Christian, donc de Stanley" $(\text { entretien })^{11}$. Chez ce lecteur, l'identification avec l'auteur est extrêmement forte

${ }^{11}$ Les sources des extraits de discours d'élève seront mentionnées comme suit : "récit " indique le récit de la première lecture, "texte final " indique le texte réflexif rédigé à la fin de la séquence didactique, et « entretien " désigne la transcription de l'entretien d'explicitation. 
et structure tout le parcours interprétatif: Lucas se sent proche de l'auteur, qu'il appelle familièrement par son prénom; il explique qu'au fil de sa lecture il a perçu " des similitudes entre l'auteur et [lui]-même ", comme si ce dernier "était devenu un proche ou un ami ", allant même jusqu'à le contacter directement via Facebook. L'entretien nous a permis de comprendre que ce fort investissement subjectif sur la personne de l'auteur passe par une identification avec le personnage qui repose sur l'expérience personnelle de la discrimination raciste que Lucas a subie à l'école. Cette double identification n'est pas le fait d'une confusion entre deux notions, puisqu'elle fait l'objet d'un processus réflexif: " moi je m'étais mis dans la peau de Christian, c'est comme si je m’identifiais dans la peau de Stanley, enfin, en faisant l'hypothèse que Stanley ait vécu ce qu'a vécu Christian ${ }^{12}$ (entretien de Lucas). Dans le récit d'un autre élève (Normand), la volonté d'attribuer au texte une valeur autobiographique est délibérée et explicitement liée à l'affectivité du lecteur: "Ce récit est aussi un texte avec un aspect autobiographique - ce qui renforce les émotions ressenties -, car la narratrice (Évelyne) et Christian sont d'origine haïtienne, mais vivent au Québec, tout comme l'auteur, Stanley Péan, qui est un Haïtien venu s'installer dans sa jeunesse au Québec » (récit).

Le fait que les élèves attribuent spontanément de nombreuses significations à une instance auctoriale devrait nous inviter à considérer «l'auteur impliqué " comme une catégorie à part entière dans une théorie de la lecture subjective.

Le lecteur pressent le rôle [de l'auteur impliqué] dans la mesure où il appréhende intuitivement l'œuvre comme une totalité unifiée. Spontanément, il ne rapporte pas seulement cette unification aux règles de composition, mais aux choix et aux normes qui font précisément du texte l'œuvre d'un énonciateur, donc une œuvre produite par une personne et non par la nature. (Ricœur 1985 : 292)

Le fait d'attribuer des énoncés à l'auteur impliqué résulterait d'un effort de compréhension qui vise à reconfigurer l'unité de l'œuvre intuitivement perçue. En cela, il s'agirait peut-être d'une des premières réalisations observables de la compréhension subjective de l'œuvre. Or, plusieurs défis se posent quant à la formation des sujets lecteurs.

Premièrement, l'auteur impliqué est un déguisement de l'auteur réel, lequel disparait en se faisant voix narrative (Ricœur 1985 : 310), ce qui constitue d'ailleurs une composante essentielle de la stratégie de persuasion du narrateur. Si l'on souhaite donner aux lecteurs en formation la responsabilité qui leur incombe dans la refiguration de l'œuvre, il faudrait les amener à saisir le rôle de l'auteur impliqué dans cette stratégie de persuasion. Cependant cet objectif peut s'avérer

${ }^{12}$ Les discours d'élève sont transcrits sans correction linguistique de notre part. 
difficile à mettre en œuvre du fait que les élèves amalgament souvent l'auteur impliqué et l'auteur réel. De plus, nous avons constaté dans les discours d'élève la prégnance de représentations héritées de l'enseignement traditionnel de la lecture, très fortement marqué par l'historicisme lansonnien, en particulier par la recherche de liens de causalité entre la vie et l'œuvre de l'auteur. Or, dans les représentations comme dans les pratiques de la lecture à l'école, la survalorisation de l'intention de l'auteur, comme unique dépositaire du sens de l'œuvre, a pour corolaire l'insuffisance constitutive du lecteur, en particulier de l'élève. Le défi pour l'enseignant consisterait à accueillir le premier mouvement spontané de la compréhension, qui repose sur la recherche d'une synthèse des significations intuitivement attribuées à l'auteur impliqué, puis, à amener les élèves à considérer les diverses instances narratives et donc l'éclatement de l'unité du sens. Renoncer à l'identité posée entre la totalité du sens et l'unicité de l'intention auctoriale constitue une démarche peu sécurisante, mais qui semble nécessaire à la formation de sujets lecteurs réflexifs et critiques. Deuxièmement, il importe de considérer que le narrateur, qu'il soit digne ou non de confiance, permet de faire varier la distance entre l'auteur impliqué et les personnages. Selon Ricour, " un degré de complexité est du même coup induit chez le lecteur, complexité qui est la source de sa liberté face à l'autorité que la fiction reçoit de son auteur » (1985: 294). Amener les lecteurs en formation à considérer les écarts de points de vue entre les différentes instances (auteur impliqué, narrateur et personnages) pourrait être un moyen de revaloriser leur rôle dans l'élaboration des interprétations.

\subsubsection{Certaines instances narratives sont-elles privilégiées par les lecteurs en formation?}

Dans les textes rédigés après la première lecture de la nouvelle, la plupart des élèves privilégient une instance (la narratrice ou le personnage principal), c'est-à-dire qu'ils concentrent leurs efforts sur l'attribution de significations aux actions ou aux discours d'un personnage en particulier. Le fait de privilégier une instance narrative apparait comme une étape nécessaire de la lecture en cela qu'il témoigne de l'investissement de ressources subjectives nombreuses, notamment pour inférer les motivations des personnages à partir des dialogues et des actions narrées. Progressivement, la relecture et les discussions entre pairs amènent les élèves à prendre en compte différentes instances. Attribuer des motivations à différents personnages leur permet d'identifier des points de vue variables et parfois contradictoires sur les évènements narrés. Or, essayer de concilier (provisoirement et partiellement) ces différents points de vue pour dégager une cohérence de l'intrigue favorise la prise de distance avec le texte lu. C'est le cas de Manon qui décrit en ses mots les divers points de vue des personnages qu'elle a adoptés : 
Manon $^{13}$ : J'me mets à [la] place [des personnages] et puis je reviens à ma place et j'm'écarte.

I : Donc, en fait, tu te mets à leur place, tout en restant toi ?

Manon: Oui. Parce que dans l'histoire je me suis mis à la place d'Évelyne, enfin j'ai essayé de la cerner, dans la place d'Évelyne, pourquoi elle avait besoin de Christian, et puis, j'me suis mis aussi à la place d'Edgar, qu'il avait raison, après je suis redevenue moi et j'me suis dit que tous les deux avaient des arguments compatibles, enfin des bons arguments. Et que voilà j'en ai pas placé un au-dessus de l'autre. (Entretien)

La capacité de discerner différentes instances et de leur attribuer des énoncés parfois contradictoires relève autant d'une prise de distance avec le texte que de la mise à distance réflexive de la lecture elle-même. Ainsi Manon est-elle capable d'expliciter en quoi la prise en compte de différentes voix narratives caractérise sa préférence pour une posture de lecture distanciée.

Manon : Je préfère rester à distance parce que si on rentre à fond dans l'histoire on est sur un personnage, on ne voit que son point de vue, tandis qu'à distance on voit tout ce qui se passe, tous les p'tits, les arrière-plans, tout c'qui ont des idées qui veulent menacer le personnage principal. À distance... À distance, on voit mieux, c'est comme si on se rapproche d'une personne [elle fait un cadre avec ses mains et mime le zoom d'une caméra] on voit que ses yeux. Si on s'éloigne, on voit tout son visage. On voit mieux à distance que plutôt quand on est dans le personnage. (Entretien)

Le développement de l'activité réflexive repose en partie sur la capacité des lecteurs de mettre à distance diverses voix narratives, au fur et à mesure que le parcours interprétatif progresse. Paul Ricœur emprunte à Iser le concept de " point de vue voyageur " pour rendre compte de ce double mouvement de diversification et de transformation. «Le concept de point de vue voyageur [...] exprime le double fait que le tout du texte ne peut jamais être perçu à la fois ; et que, situés nous-mêmes à l'intérieur du texte littéraire, nous voyageons avec lui au fur et à mesure que notre lecture avance » (Ricœur 1985 : 306).

Les élèves avec lesquels nous avons travaillé ont eu tendance à privilégier spontanément une voix narrative au détriment des autres, en particulier lorsque celle-ci se rapproche, à leurs yeux, de l'intention présumée de l'auteur. Toutefois, au fur et à mesure des discussions et des relectures, ils ont considéré diverses instances narratives auxquelles ils ont attribué des énoncés, même si ces derniers semblaient contradictoires. Ce processus d'attribution est fortement corrélé à la

\footnotetext{
${ }^{13}$ Les discours et textes d'élève sont retranscrits sans correction linguistique de notre part.
}

\section{Arborescences}


mobilisation de ressources subjectives. Parmi les ressources de nature axiologique, quelles sont celles qui ont été observées dans les discours d'élève? Ces ressources influencent-elles les significations attribuées aux diverses voix narratives?

\subsection{Les ressources axiologiques mobilisées pour interpréter : le cas de Juliette}

Dans les discours oraux et écrits des élèves, nous avons observé trois catégories de ressources axiologiques: la verbalisation de prise de position idéologique, la formulation de jugements moraux à l'encontre des personnages et la recherche de modèles de comportement.

Nous présentons ici le parcours de Juliette, une élève de la classe de seconde à Toulouse. Elle éprouve certaines difficultés en lecture; dans son premier récit de lecture, elle énonce son appréciation de la nouvelle :

J'ai aimé cette nouvele car il parle des différentes cultures, du racisme et de l'amour entre une femme et un petit garçon. Il y a des passages que je n'ai pas aimé comme, par exemple, les moments où Christian Marcellin se fait frotter la peau par ses camarades, le moment où il se trouve dans le containeur, en pleurs, en sang, où il avait été rossé, plein d'urine puis balancé à la poubelle. Je n'apprécie pas ces moments parce que juste comme il a une couleur de peau différente, les autres élèves lui font du mal. (Récit de Juliette)

L'entretien d'explicitation portant sur ce texte révèlera que le fait que la violence raciste ait retenu l'attention de Juliette provient à la fois d'une expérience personnelle de la discrimination à l'école et d'une prise de position idéologique antiraciste. Toutefois, peu d'élèves ont explicité aussi clairement des présupposés idéologiques. Par contre, nombreux sont ceux qui ont exprimé des jugements moraux sur les personnages. En voici un exemple dans la suite du récit de Juliette :

\begin{abstract}
Ensuite, j'ai aimé l'amitié qui nait entre Évelyne Lhérisson et Christian Marcellin. Plus on s'approche de la fin, plus on se rend compte que la femme aime le petit garçon comme si c'était son fils et lui aussi comme si c'était sa mère. Par contre, je n'ai pas compris pourquoi le père négligeait ses origines et disait à son fils qu'il n'était pas haïtien, mais québécois. Je pense qu'au contraire il devrait être fier et transmettre à son fils cette culture. (Récit de Juliette)
\end{abstract}

Après la première lecture, la formulation d'un jugement de gout par cette lectrice repose davantage sur l'évaluation morale des actions des personnages que sur une appréciation critique de l'œuvre. Dans l'entretien d'explicitation, l'influence de ces 
ressources axiologiques sur la compréhension des relations entre les personnages est encore plus évidente :

Juliette : Après... j'ai compris le texte comme ça là. Peut-être que j'ai mal compris le texte aussi, mais... [...] oui, Évelyne elle rêve/déjà elle a perdu sa poupée. Et moi, j'ai l'impression qu'elle comble ce manque avec Christian. Mais Christian ce n'est PAS son enfant. Et je trouve qu'elle s'imagine tellement de choses comme si c'était son fils, mais [...] ça peut être bien pour Christian, mais ça peut être mal aussi parce que, du coup, ses propres parents, il va peut-être moins les aimer que ELLE. Donc du coup, c'est pour ça que j'ai écrit ça, parce que, c'est bien, il faut toujours croire en ses rêves [...] Mais il faut savoir faire le bien et le mal. De ce que ça peut apporter. [...]

I : Peut-être que ça détruit Christian, peut-être que...

Juliette : Ou peut-être l'amour ou le lien qu'il avait avec son père, parce que $[\ldots]$ c'est elle qui lui apporte plein d'informations sur Haïti et son père qui lui apporte rien du tout. (Entretien)

On voit ici comment la formulation de valeurs, de jugements moraux et la recherche de comportements exemplaires soutiennent l'attribution de significations aux motivations et aux conséquences des actions des personnages. En ce sens, la mobilisation de ressources axiologiques peut contribuer à la compréhension du texte. Mais, est-ce toujours le cas ?

Dès la première lecture, Juliette a exprimé une incompréhension face au fait que "le père [Alceste] négligeait ses origines". Cette incompréhension ne provient pas d'une lacune de type cognitif, comme l'absence de stratégies de lecture, par exemple. Elle repose plutôt sur un hiatus entre, d'une part, la figure de migrant assimilé à la culture d'accueil représentée dans le texte et, d'autre part, la valorisation de l'appartenance à une communauté étrangère que Juliette défend. Entre le reniement de la culture haïtienne du personnage et la prégnance d'un sentiment d'appartenance à la culture centre-africaine de la lectrice, il y a une contradiction que Juliette n'aura de cesse d'explorer. La confrontation des systèmes de valeurs est si forte que, dans l'entretien, Juliette n'est pas capable de justifier son jugement autrement qu'en réaffirmant ses propres valeurs.

Juliette : À un moment, le père, il dit à Christian qu'il est Québécois. Et, le père, il est bien Haïtien, si j'ai bien compris le texte?

I : Oui.

Juliette : Ça, c'est un truc que je... on dirait qu'il repousse, enfin, qu'il refuse ses origines. Et c'est un truc que, j'sais pas, il devrait être fier, au contraire, quoi ! [...]

I : Pourquoi est-ce qu'il devrait être fier, Alceste? 
Juliette : Ben parce que! C'est, enfin je sais pas, comment dire... C'est ses origines, c'est lui, c'est... Ben je sais pas comment l'dire. Ben, moi personnellement, je suis fière de mes origines. (Entretien)

La prédominance de ses ressources subjectives (notamment ses appartenances socioculturelles et ses valeurs) a fait écran à une compréhension plus nuancée de la thématique de la dislocation identitaire. Dans l'entretien, nous avons relu ensemble l'épilogue. La relecture a permis à Juliette de se décentrer pour comprendre les motivations du père de Christian sous un jour nouveau :

Juliette relit : "J'avais peut-être jugé trop sévèrement Alceste Marcellin. $\mathrm{Au}$ fond, il aimait son fils, de cet amour sincère et presque douloureux que bien des pères, par fausse fierté masculine ou par timidité, n'osent pas exprimer " [elle s'interrompt] Ça contredit ce que j'ai dit.

I : Qu'est-ce qu'on apprend dans cette fin ? [...]

Juliette: Euh... Ben on apprend déjà que Évelyne, elle a jugé trop sévèrement. Que finalement, eh bien, comment on dit, il rejetait pas ses origines, mais pourquoi il les montrait pas alors? Pourquoi il voulait pas... pourquoi il lui racontait pas?

I : À ton avis ? Est-ce que tu peux proposer une hypothèse ? [...] Pourquoi il aurait voulu que Christian soit un petit Québécois et pas un petit Haïtien?

Juliette : Ben, parce ça aurait été mieux pour lui [...] pour Christian [...] pour dans la société, ça aurait plus facile pour lui, de se trouver peutêtre un boulot, un travail. Et puis d'avoir la nationalité québécoise, oui, ouais, pour ça. Pour la vie, en fait. (Entretien)

La formation du sujet lecteur implique de le guider vers ce moment critique où il prend conscience de ses contradictions et met à distance ses interprétations. Pour Juliette, force est de constater que ce processus réflexif a constitué un défi majeur. Après avoir vu que la prise en compte de diverses voix narratives favorisait la mise à distance du texte, nous observons ici une autre composante de l'activité réflexive, à savoir la mise à distance des interprétations. Il semble que ce soient les détours par l'altérité textuelle (par la relecture) et par l'autre lecteur du texte (ici la chercheuse) qui favorisent le plus cette distanciation de soi à soi. Une troisième composante de l'activité réflexive consiste pour le lecteur à mettre à distance non seulement son interprétation, mais les ressources qui influencent cette interprétation. 


\subsection{La mise à distance des ressources axiologiques par les lecteurs et le développement de leur réflexivité}

Mélissa et Cyril sont deux élèves québécois du deuxième cours de littérature au collégial. Leurs discours nous permettront d'illustrer comment les élèves mettent à distance les ressources axiologiques qu'ils ont mobilisées pour interpréter le texte et dans quelle mesure ce retour sur soi-même comme lecteur contribue au développement de leurs habiletés interprétatives.

\subsubsection{De la recherche d'un comportement à la remise en question d'une doxa : le cas de Mélissa}

Dans son texte final, Mélissa, une élève plutôt discrète en classe, a rendu compte de manière intime des questionnements éthiques que la lecture de la nouvelle a suscités chez elle. L'extrait suivant illustre comment cette lectrice a établi des liens explicites entre certaines de ses ressources axiologiques, ici la recherche de modèles de comportement, et son interprétation des motivations et des actions du personnage de Christian :

Tout comme Christian je me sens parfois perdue dans le monde autour de moi. De nombreuses questions se posent à moi et j'essaie toujours d'y obtenir des réponses. De plus, lorsque des choses ne marchent pas comme je le voudrais et que tout va mal, je m'invente moi aussi un monde imaginaire où tout irait mieux. À sa place, j'aurais agi comme lui. J'aurai essayé d'en savoir le plus possible sur ma culture d'origine. (Texte final de Mélissa)

Lorsqu'elle écrit "à sa place, j'aurais agi comme lui ", Mélissa n'exprime pas seulement son identification avec le personnage, mais une expérience propre à la lecture littéraire, qui consiste à élaborer des significations en se projetant dans des scénarios fictionnels. Or, ce qui est en jeu dans cette variation imaginative du sujet, c'est le rapport parfois conflictuel à l'origine culturelle. Par la suite, elle s'interroge sur les motivations d'un autre personnage: Alceste, le père de Christian.

Dans le texte, le père de Christian, en immigrant au Québec, décide d'oublier et de faire taire ses origines. Je ne comprends pas pour quelles raisons il agit ainsi. Bien souvent, c'est le contraire qui se produit lorsque des gens immigrent au Québec. Ils désirent continuer de vivre avec leurs habitudes de vie de leurs pays d'origine. Je me demande donc si beaucoup de personnes venues s'établir ici décident de vivre comme le père de Christian. (Texte final) 
Mélissa rend compte explicitement de la contradiction entre la figure de l'immigrant "assimilé " représentée dans le texte et ses propres représentations, contradiction influencée par une doxa, véhiculée notamment par les médias à propos du débat sur les accommodements raisonnables ${ }^{14}$.

\begin{abstract}
Mélissa : Je me demandais pourquoi que le monde agissait comme ça, mais j'avais, j'avais jamais vraiment pensé qu'il y avait du monde qui venait ici, puis qui disait : OK, je deviens BLANC. J'pensais pas que ça se pouvait vraiment là. Mais t'sais, je m'étais jamais posé cette questionlà. D'après moi, on entend tout le temps le monde qui immigre, t'sais c'est... avec les accommodements raisonnables. "Je veux garder mon voile" puis tout là. J'pensais pas qu'il y a du monde qui se disait: "J'deviens blanc puis, euh... j'oublie toute mon ancienne culture", parce que t'sais, ça fait quand même partie d'eux là, ça doit être quelque chose difficile qui t'fasse changer [...] Bien, Christian, lui, justement, j'trouve que c'est important de garder ta culture d'origine. (Entretien)
\end{abstract}

Cet extrait illustre le fait que le récit littéraire constitue un formidable réservoir de propositions éthiques susceptibles de remettre en question la vision du monde du lecteur. Selon Ricœur, «la stratégie de persuasion fomentée par le narrateur vise à imposer au lecteur une vision du monde qui n'est jamais éthiquement neutre, mais qui plutôt induit implicitement ou explicitement une nouvelle évaluation du monde et du lecteur lui-même. [...] il reste qu'il appartient au lecteur, redevenu agent, initiateur d'action, de choisir entre les multiples propositions de justesse éthique véhiculées par la lecture" (Ricœur 1986: 446). La mise à distance des ressources axiologiques que permettent l'écriture réflexive et l'entretien d'explicitation nous semble une composante essentielle du développement des compétences interprétatives des jeunes lecteurs, d'autant plus que ces derniers sont confrontés à des modèles culturels très divergents et souvent produits hors de l'école. En ce sens, la lecture littéraire offre une médiation privilégiée pour mettre à

\footnotetext{
${ }^{14}$ L'expression accommodement raisonnable renvoie à une obligation juridique découlant du droit canadien à l'égalité, applicable dans une situation de discrimination, et consistant à aménager une norme, en accordant un traitement différentiel à une personne qui, autrement, serait pénalisée par l'application d'une telle norme. Selon Pierre Bosset, professeur en sciences juridiques à l'UQAM, « il est erroné d'assimiler accommodement raisonnable et immigration [...] le concept d'accommodement raisonnable est applicable à une très grande variété de motifs de discrimination, parmi lesquels figurent le handicap, le sexe, la grossesse, l'âge et l'origine nationale» (dans Toussaint 2010 : 68). La notion d'accommodement raisonnable a fait l'objet d'une surenchère médiatique et d'une récupération politique tendant à stigmatiser certaines communautés ethniques ou religieuses. Par exemple, au cours des années 2008 et 2009, la question du port du voile intégral, au moment d'une élection provinciale, a suscité de nombreux débats sur l'identité au Québec.
} 
distance de manière critique les normes et les valeurs plus ou moins partagées par une communauté socioculturelle.

\title{
5.3.2. De la mise à distance critique des valeurs collectives à la prise de conscience de leur influence sur I'interprétation : le cas de Cyril
}

Terminons cette analyse en nous penchant sur le discours de Cyril concernant la diversité des interprétations produites par ses pairs. Il a supputé que des systèmes de valeurs différents pouvaient expliquer des divergences d'interprétation. Dans l'entretien, nous sommes revenus sur les jugements moraux formulés par des élèves de sa classe au sujet de la relation fusionnelle entre Évelyne et Christian.

\begin{abstract}
I : [Certains] la jugent sévèrement, disent que [la narratrice] s'accapare un enfant qui n'est pas le sien...

Cyril : [...] T'sais c'est l'opinion de chacun là. Vu que c'est un texte, l'auteur a le droit de (rire) t'sais de faire faire tout, tout ce qu'il veut à Évelyne [...] j'me suis dit: si elle est comme ça dans vie puis si les parents sont d'accord [...] c'est sûr que c'est bizarre là, mais, parce que t'sais, nous autres, notre culture [...], on protège nos enfants puis, on est comme ça ici là, mais y a des, y a d'autres cultures surement dans le monde que $[. .$.$] tu peux genre laisser tes enfants. En tout cas de nos$ jours [...] les parents surprotègent leurs enfants là. Fait que t'sais, moi j'ai toujours été pas mal libre chez nous là, fait que j'ai pas pensé, j'ai j'ai pas dit : ah c'est c'est révoltant puis tout ça là. J'trouvais ça normal là si les parents sont d'accord puis que le fils et Évelyne sont bien làdedans. (Entretien)
\end{abstract}

Cyril se présente comme un sujet lecteur situé dans une communauté culturelle ("nous autres [...] notre culture») qui véhicule des valeurs susceptibles d'influencer le cadre interprétatif commun. De plus, il conçoit que les appartenances à d'autres cultures ("y a d'autres cultures surement dans le monde») produisent des variations axiologiques qui influencent les cadres interprétatifs. En dépit des maladresses d'expression, cet extrait témoigne d'un effort remarquable du sujet pour concevoir la production des diversités interprétatives. Cette conceptualisation repose sur une généralisation de type inductif: le sujet lecteur (l'individu) se situe dans une communauté de lecteurs (la classe), elle-même située dans une communauté culturelle (le Québec) qui s'inscrit dans un contexte transculturel (le monde). On peut penser que le cadre concret de l'échange intersubjectif sert de base à la réflexion heuristique sur le rapport à autrui, par généralisation successive. Autrui s'avère un médiateur de la distanciation de soi à soi, puisque Cyril finit par dégager l'influence de ses propres 
ressources axiologiques sur son interprétation. Tout en se situant explicitement dans un contexte culturel, il développe son esprit critique en signalant qu'il n'en partage pas forcément toutes les valeurs.

Cyril ne se contente pas de constater la présence des diversités interprétatives, il tente d'expliquer le processus de l'interprétation subjective :

Cyril : [dans le texte] Y a tellement de détails puis d'éléments qui sont pas tellement expliqués que tout le monde, par rapport à ce qu'ils ont déjà vécu, leurs expériences, leurs valeurs, puis tout ça là. Puis vu qu’il y a personne qui est pareil, ben il y a personne qui comprend les choses pareillement là. (Entretien)

Il met au jour le rapport entre l'incomplétude du texte et la dimension subjective de l'activité lectorale. Il dégage même plusieurs ressources de la subjectivité : le vécu, l'expérience, les valeurs. À partir de la lecture de La plage des songes, il est parvenu à formuler une conception de la lecture subjective, puisqu'il est capable d'identifier les liens entre la diversité des ressources subjectives et les éléments textuels qui suscitent l'engagement des lecteurs : l'intrigue, les personnages, les valeurs qui leur sont attribuées.

I : Est-ce que tu penses que c'est ce texte-là ou c'est généralisable à d'autres textes?

Cyril : Non, c'est sûr que c'est généralisable là. Parce que t’sais, tant qu'il y a une intrigue, qu'il y a des des personnages avec des des valeurs différentes puis tout ça là, ça va être euh, tout le monde va comprendre des affaires différentes là. [...] Puis de toute façon, les auteurs de ces textes-là ils veulent pas que tu comprennes ce que eux autres ils veulent dire, là, ils veulent que tu te fasses ta propre interprétation du texte. (Entretien)

Au terme de son parcours de lecteur, Cyril a démystifié la recherche d'un sens univoque dont l'auteur serait l'unique dépositaire. Il s'est affirmé comme un sujet lecteur réflexif, capable de produire ses propres interprétations.

\section{Conclusion}

Si les sujets lecteurs privilégient spontanément une instance énonciative, notamment celle de l'auteur, les discussions entre pairs et les relectures semblent favoriser la prise en compte progressive des écarts de point de vue entre diverses instances narratives. Or, l'attribution de significations à diverses voix narratives semble corrélée à la mobilisation de nombreuses ressources, en particulier, des ressources axiologiques. Dans les discours d'élève, nous avons observé des jugements moraux sur les personnages, la recherche de comportements 
exemplaires, la verbalisation de présupposés idéologiques. Dans plusieurs cas, ces ressources contribuent de manière significative à la formulation et à la résolution de problèmes interprétatifs par les élèves. Toutefois, dans d'autres cas, elles peuvent entraver une interprétation nuancée de la polysémie textuelle, surtout si elles sont mobilisées de manière exclusive et implicite. Le lecteur peut se trouver pris au piège du solipsisme interprétatif. Le développement de la réflexivité, qui favorise le moment critique, apparait alors comme le complément nécessaire de la prise en compte de l'investissement subjectif.

L'activité réflexive repose à la fois sur la mise à distance du texte (soutenue, en particulier, par la prise de conscience de l'hétérogénéité énonciative) et sur une "distanciation de soi à soi " en tant que sujet lecteur. Dans les discours des élèves, nous avons dégagé certaines composantes de la réflexivité: la mise à distance des interprétations, l'explication des ressources ayant influencé ces interprétations, la reformulation critique des valeurs ou des opinions partagées au sein d'une communauté. L'activité réflexive parait particulièrement significative lorsqu' elle aboutit au "moment critique " (Ricœur 1986 : 131) de la compréhension de soi. Ce moment critique est celui où le lecteur prend conscience des ressources subjectives qui influencent la compréhension concomitante du sens et de soi. Les sujets lecteurs participant à notre recherche ont fait preuve de capacités interprétatives et réflexives qui paraissent sous-exploitées dans l'enseignement actuel de la lecture littéraire. En contexte de formation, la prise de conscience des idéologies, des valeurs, des modèles de comportement qui influencent l'activité interprétative semble nécessiter un accompagnement didactique, dont les modalités restent encore largement à formaliser.

\section{Références bibliographiques}

Bhabha, H. 2007. Les lieux de la culture : une théorie postcoloniale. Traduit par F. Bouillot. Paris : Payot. [1993].

Caillois, R. "Fantastique». Dans Encyclopedia Universalis. URL: http://www.universalisedu.com/encyclopedie/fantastique/. Page consultée le 21 mai 2015.

Citton, Y. 2007. Lire, interpréter, actualiser. Pourquoi les études littéraires? Paris : Amsterdam.

Daunay, B. 2007. "État des recherches en didactique de la littérature, Note de synthèse ». Revue française de pédagogie, 159: 139-189.

Daunay, B., I. Delcambre, et Y. Reuter, dir. 2009. Didactique du français, le socioculturel en question. Villeneuve-D’Ascq : Presses de l'Université du Septentrion.

Dufays, J.-L. 2007. «Le pluriel des réceptions effectives. Débats théoriques et enjeux didactiques ». Recherches 46 (1): 71-90.

Dufays, J.L. 2013. "Sujet lecteur et lecture littéraire: quelles modélisations pour quels enjeux? ». Recherches et travaux 83. URL: http://recherchestravaux.revues.org/666. Page consultée le 7 janvier 2016.

Dufays, J.-L., Gemenne, L. et Ledur D. 2005. Pour une lecture littéraire, histoire, théories, pistes pour la classe. Bruxelles : De Boeck. 
Eco, U. 1985. Lector in fabula. Paris : Grasset. [1979].

Étienne, G. 1974. Le nègre crucifié. Montréal : Nouvelle optique.

Fish, S. 2009. Quand lire c'est faire. L'autorité des communautés interprétatives (3e édition). Traduit par É. Dobesnesque. Paris : Les prairies ordinaires. [1980].

Foucault, M. 2009. Le corps utopique, les hétérotopies. Clamecy, France: Nouvelles Éditions Lignes.

Gervais, B. et Bouvet R. 2007. Théories et pratiques de la lecture littéraire. Québec: Presses Universitaires du Québec.

Giasson, J. 1990. La compréhension en lecture. Québec: Gaétan Morin.

Glissant, É. 1990. Poétique de la Relation, III. Paris : Gallimard.

Iser, W. 1971. "Indeterminacy as the Reader's Response in Prose Fiction." Dans Aspects of Narrative sous la direction de J.H. Miller. Traduit par R. Ingarden. New York: Columbia University Press : 1-45.

Iser, W. 1985. L'acte de lecture. Théorie de l'effet esthétique. Bruxelles : Pierre Mardaga. [1976].

Laferrière, D. 2007. Pays sans chapeau. Monaco : Le Rocher. [1997].

Laferrière, D. 2009. L'énigme du retour. Paris : Grasset.

Lahire, B. 2004. La Culture des individus : dissonances culturelles et distinction de soi. Paris : La Découverte.

Langlade, G. et Fourtanier, M.-J. 2007. "La question du sujet lecteur en didactique de la lecture littéraire ". Dans La didactique du français. Les voies actuelles de la recherche, sous la direction d'É. Falardeau, C. Fisher, C. Simard et N. Sorin. Québec: Presses de l'Université Laval : 101-121.

Louichon, B. 2009. La littérature après coup. Rennes: Presses Universitaires de Rennes.

Louichon, B. 2011. "La lecture littéraire est-elle un concept didactique ? ». Dans Les concepts et les méthodes en didactique du français, sous la direction de B. Daunay, Y. Reuter et B. Schneuwly. Namur : Presses universitaires de Namur : 195-214.

Mazauric, C., Fourtanier, M.-J. et Langlade, G. dir. 2011a. Le texte du lecteur. Bruxelles : Peter Lang.

Mazauric, C., Fourtanier, M.-J. et Langlade, G. dir. 2011b. Textes de lecteurs en formation. Bruxelles : Peter Lang.

Miles, M.B. et Huberman, A.M. 2003. Analyse des données qualitatives. Traduit par M. Hlady Rispal. Bruxelles : De Boeck. [1994].

Olivier, N. 1998. "Stanley Péan: l'œuvre au noir». Lettres québécoises: la revue de l'actualité littéraire 90 : 8-10. URL : http://id.erudit.org/iderudit/38047ac. Page consultée le 21 mai 2015.

Ollivier, É. 1977. Paysage de l'aveugle. Montréal : Pierre Tisseyre.

Ollivier, É. 1994. Passages. Paris : Le serpent à plumes.

Péan, S. 1998. La plage des songes et autres récits d'exil. Montréal : Bibliothèque Québécoise. [1988].

Phelps, A. 1976. Mémoire en colin-maillard. Canada, Montréal : Nouvelle optique.

Reuter, Y. 1995. "La lecture littéraire : éléments de définition ». Le Français Aujourd'hui 112 : 70-78.

Ricœur, P. 1985. Temps et Récit III, Le temps raconté. Paris : Seuil, Points.

Ricour, P. 1986. Du texte à l'action II, Essais d'herméneutique. Paris : Seuil, Points.

Ricœur, P. 1990. Soi-même comme un autre, Paris : Seuil, Points.

Rouxel, A. 2002. "Qu'entend-on par lecture littéraire?». Dans La lecture et la culture littéraires au cycle des approfondissements, sous la direction de C. Tauveron. Paris: DESCO et CRDP de Versailles.

\section{Arborescences}

Revue d'études françaises

ISSN: 1925-5357 
URL : http://eduscol.education.fr/D0126/lecture_litteraire_rouxel.htm. Page consultée le 7 janvier 2016.

Rouxel, A. et Langlade, G. dir. 2004. Le sujet lecteur, lecture subjective et enseignement de la littérature. Rennes, France: Presses Universitaires de Rennes.

Sauvaire, M. 2013. Diversité des lectures littéraires. Comment former des sujets lecteurs divers? Thèse de doctorat. Québec et Toulouse : Université Laval et Université Toulouse-le-Mirail.

Sauvaire, M. 2015. "La diversité des sujets lecteurs dans l'enseignement de la lecture littéraire». Éducation et didactique, 9 (2) : 21-31.

Simard, C., Dufays, J.-L., Dolz, J. et Garcia-Debanc, C. (2010). Didactique du français langue première. Bruxelles : Éditions De Boeck.

Tauveron, C., dir. 2002. La lecture et la culture littéraires au cycle des approfondissements. Actes de la DESCO. URL : http://eduscol.education.fr/cid46330/actes-de-l-universite-d-automnela-lecture-et-la-culture-litteraires-au-cycle-des-approfondissements.html. Page consultée le 7 janvier 2016.

Todorov, T. 1970. Introduction à la littérature fantastique. Paris : Seuil.

Toussaint, P. 2010. La diversité ethnoculturelle en éducation, enjeux et défis pour l'école québécoise. Montréal : Presses de l'Université du Québec.

Vermersch, P. 1991. "L'entretien d'explicitation ». Les cahiers de Beaumont 53 : 63-67.

Vermersch, P. 2004. "Aide à l'explicitation et retour réflexif ». Éducation Permanente 160 : 71 80 . 